\title{
QUANTUM CURVATURE: TOWARD THE UNITY OF RELATIVITY AND QUANTUM MECHANICS
}

\author{
Wu Xinzhong* \\ *Shanghai Jiaotong University, School of History and Culture of Science E-mail: sju@ sina.com
}

*Corresponding Author: -

E-mail: sju@sina.com

\begin{abstract}
: -
Quantum curvature interpretation has greatly promoted the unification of relativity and quantum mechanics in physics thought level. In this paper, we discuss the deep unification of relativity and quantum mechanics according to new research by some scholars. This paper involves the derivition of quantum hypothesis from the relativistic transformation, the relation between Compton's matter wave and De Broglie's matter wave, and the quantum divition mechanism of inertial systems in the superposition principle of quantum states.
\end{abstract}

Keywords: Quantum Curvature, Compton matter wave, Dual 4-dimensinal space-time.

Fund project: Humanity and Social Science general research project in the Chinese Ministry of Education: Philosophy research in relation between Being and Information in quantum computing and thermodynamics, Serial number:14YJA720004.

\section{(c) $(\$)$}




\section{Quantum hypothesis and relativistic transformation}

Quantum theory originated from the anomalous empirical problems in classical physics, as well as the conceptual problems associated with them. The empirical problems are black body radiation, photoelectric effect, atomic spectrum, atomic stability, specific heat and so on. The concept problems focus on the heat balance between discrete particle system and continuous field system, various problems and paradoxex between themal radiations and material structure. The particle program of Newtonian mechanics and the field theory of electromagnetics cannot be communicated and coordinated by means of thermodynamics and statistical mechanics, this exposes the deep crisis of classical physics, which is the historical background of the quantum theory revolution.

In 1859, it had been proved that the component of the blackbody radiation (energy distribution in frequency) depends only on the temperature of the surrounding cavity wall and does not depend on the material of the cavity wall according to the study of Kirchhoff and others. In other words, when radiation is at the same wavelength at the same temperature, the emissivity to absorptivity ratio is the same for all objects. What is involved here is a certain temperature blackbody, electromagnetic radiation and particles are in equilibrium: $\mathrm{dE}=\mathrm{c} \rho(v, \mathrm{~T}) \mathrm{d} v$ (1.1) In 1900, Rayleigh and Jeans calculated that all the energy of an object will be absorbed by the field according to the energy equipartition principle -- no limit. There is an absurdity in physics here - the UV catastrophe: If the matter and radiation oscillator is completely continuous according to classical concept, molecular and atomic thermal motion energy of an object will continue to run into electromagnetic field, and produce electromagnetic radiation vabrations of higher and higher frequency, which can never reach a thermal equilibrium. Because the heat balance means needs for infinite radiation energy, and leads to the so-called 'UV catastrophe': $\rho(v, T)=\left(8 \pi v^{2} / \mathrm{c}^{3}\right) \mathrm{kT}$

Another Wien formula is consistent with experimental facts in the short wavelength , but there is a great error in the long wavelength:

$$
\rho=\left(8 \pi v^{3} / c^{3}\right) a^{\prime} e^{-a v / T}
$$

The a and a' in the formula are two constants[1, p17].

The radiation in the cavity field is considered as a standing wave collection of various discrete wavelengths in the derivation of the Wien formula, each possible standing wave corresponds to a degree of freedom of the electromagnetic field .Then, Wien introduced a novel hypothesis: 'When gas in T temperature is in equilibrium with radiations, the wavelength and intensity of radiation released from molecules depend only on the molecular velocity'. This introduces a hypothesis that is incompatible with electrodynamics, so that physics is brought directly to the gate of quantum physics. In 1900, Planck adopted two ad hoc thermodynamic assumptions, and deduced the Planch law of energy frequency distribution of blackbody radiation consistent with the experiment. It accords with Rayleigh-Jeans formula in long wavelength (low temperature), and accords with Wien formula in short wavelength (high temperature):

$$
\rho=\left(8 \pi v^{2} / \mathrm{c}^{3}\right)(\mathrm{h} v / \exp [\mathrm{h} v / \mathrm{kT}]-1)
$$

The two hypothesis are as follows: One is quantum hypothesis, namely the total energy of a harmonic oscillator system consists of finite indecomposable energy packages of $\mathrm{E}=\mathrm{h} v$; another is counting hypothesis that we regard particles as identicle particles in entropy calculation of resonators. The concept of identical particles,as like as quantum hypothesis, is a brand new concept different from classical statistics concept. Without the assumption of identical particles, we cannot deduce the Planck formula, which is the inherent requirement of the blackbody radiation law.

Physicists generally believe that the quantum hypothesis appeared before special relativity, it is not contradictory to relativity, but it is logically independent of relativity. It seems impossible and unnecessary to deduce the quantum hypothesis from relativity. However, Chinese scholar Shi-Zhiheng derived the quantum hypothesis again by combining the experimental law of black body radiation and Lorentz transformation of relativity in his some physics manuscripts(2007). This indicates deep connection between quantum hypothesis and relativity. In Shi's paper, quantum hypothesis is deduced from relativistic transformation of Doppler effect at first:

We don't presuppose Planck formula $E h(v)$ in the beginning, but only assume $E=f(v)$,so the energy exchange formula of light quantum as a particle is:

$$
E^{\prime}=\gamma\left(E-\beta C P_{x}\right),
$$

Among them, $P x=P \cos \theta=E \cos \theta / c$, so

$$
E^{\prime}=\gamma(E-\beta c P \cos \theta)=\gamma(E-\beta E \cos \theta),
$$

The solution is obtained: $E^{\prime}=\frac{1-\beta \cos \theta}{\sqrt{1-\beta^{2}}} E$,

$$
\text { Or } \frac{E}{E^{\prime}}=\frac{\sqrt{1-\beta^{2}}}{1-\beta \cos \theta}=\frac{f(v)}{f\left(v^{\prime}\right)}
$$

So $E=f(v)=h v \quad(h$ is a constant independent of a reference system $)$

Secondly, if Lorentz transformation of space-time coordinates in Einstein's 'electrodynamics of moving object' is substituted into the phase factor formula of wave functions, we obtain a formula of frequency transformation: 


$$
\omega=\omega^{\prime} \frac{\sqrt{1-\left(\frac{v}{c}\right)^{2}}}{1-\frac{v}{c} \cos \theta} \text { or } v / v^{\prime}=\frac{\sqrt{1-(v / c)^{2}}}{1-\cos \theta \frac{v}{c}}=\frac{E}{E^{\prime}}=\frac{f(v)}{f\left(v^{\prime}\right)}
$$

So $E=f(v)=h v$, and $h$ must be a constant independent of a reference system to ensure the above formula correct. From this, we can infer that the energy-frequency relation $E=f(v)$ must be a linear correlation function for a wave with light velecity $\mathrm{c}$, only from Lorentz transformation under the principle of relativity, or the zero result of Michelson - Morley experiment, or the fact that a spherical light wave in the static system becomes an ellipsoid light wave in the motion system under Lorentz transformation (We calculate the product of the light energy and the sphere volume of the unit volume to compare the energy - frequency relationship between the stationary system and the motion system). This improves the importance of the Plank's original hypothesis $E=h v$ or $E=\hbar \omega$ in quantum theory, and becomes a theorem as same as $E=m c^{2} \cdot[2, \mathrm{p} 50]$

\section{Compton matter wave}

De Broglie thought that Einstein's photon formula $E=h v$ and particle equantion $E=m c^{2}$ are universal for both photons and material particles. Therefore, in the frame of reference fixed to a static particle of $m_{0}$, the internal frequency of the periodic process is $v=m_{0} c / h$.

When a stationary observer looks at this particle moving at a speed $\mathrm{v}$, its vibration frequency will be reduced to:

$$
v_{1}=v_{0}\left(1-v^{2} / c^{2}\right)^{1 / 2}=\left(m_{0} c^{2} / h\right)\left(1-v^{2} / c^{2}\right)^{1 / 2}
$$

On the other hand, the energy of this moving particle is: $E=m_{0} c^{2} /\left(1-v^{2} / c^{2}\right)^{1 / 2}$, Its corresponding frequency is: $v_{2}=v_{0}\left(1-v^{2} / c^{2}\right)^{-1 / 2}=m_{0} c^{2} / h\left(1-v^{2} / c^{2}\right)^{1 / 2}$

Obviously, $v_{1}$ and $v_{2}$ are different.

De broglie obtained phase velocity $u=c^{2} / v$ of phase wave from frequency $v_{2}$ and wavelength $\lambda=h / m v=h\left(1-v^{2} / c^{2}\right.$ )$^{1 / 2} / \mathrm{m}_{0} \mathrm{v}$, it is different from the particle velocity $\mathrm{v}$, and far greater than the speed of light c. According to De Broglie's hypothesis, the phase wave speed is larger than the speed of light. But if the phase wave does not transmit energy and information, there will be no violation of the law of causality.

In the view of a stationary observer, there is a particle with a velocity $\mathrm{v}$ and an internal oscillation frequency $v_{1}$ that associated with a wave with phase velocity $u$ and fluctuation frequency $v_{2}$. Now, a particle can be interpreted as a microscopic clock that holds the same phase with its wave and is moving at a velocity $\mathrm{u}-\mathrm{v}$ relative to its wave. In other words, for any Galilean frame of reference, the phase of the internal clock of a moving quantum particle is equivalent to the its wave phase calculated at the same point where the particle is[1,p32-34].

In De Broglie's original phase wave theory, not only the internal oscillation frequency and quantum fluctuation frequency of the particle is inconsistent, but also the velocity of the particle is not consistent with the phase velocity of the wave. In addition, relativistic formulas and Galilean frame of reference are used in De Broglie's problem analysis. In order to solve these conceptual problems, Chinese scholar Zhao Guoqiu introduced a new quantum mechanics of Compton matter wave, and put forward a geometric comprehesion of quantum probability — curvature interpretation of quantum wavefuction[3,p311-315].

We assume that the reference frame of the stationary observer is A system, and that a moving reference frame with the particle velocity V is B system. De Broglie conceived that the matter wave of B system associated with the static particles is actually a vibration. A particle is a vibrating particle, and the matter wave in A system is the result of motions of oscillating particles. But in combination with Compton wavelength, we can completely construct a real matter wave associated with a static particle on the base of current theory.

Quantum mechanics points out that wave frequency of a static particle is $\mathrm{v}_{0}=\mathrm{m}_{0} \mathrm{c}^{2} / \mathrm{h}$. Compton wavelength associated with the static particle is $\lambda_{0}=\mathrm{h} / \mathrm{m}_{0} \mathrm{c}$.

Obviously, $v_{0}$ and $\lambda_{0}$ constitute a matter wave's frequency and wavelenghth with a static particle_Compton matter wave $\varphi_{0}$, its wave velocity is light veloity $\mathrm{c}$ :

$$
\varphi_{0}=\mathrm{a}_{0} \bullet \exp \left\{(2 \pi \mathrm{i} / \mathrm{h})\left(\mathrm{P}_{0} \cdot \mathrm{r}-\mathrm{E}_{0} \mathrm{t}\right)\right\}
$$

Where $\mathrm{r}$ is a displacement vector, which is the direction of particle motion, and let $\mathrm{P}_{0} \bullet \mathrm{r}=0, \mathrm{P}_{0}=\mathrm{m}_{0} \mathrm{c}\left(\mathrm{p}_{0}=\mathrm{m}_{0} \mathrm{c}\right), \mathrm{E}_{0}=\mathrm{m}_{0} \mathrm{c}^{2}$, $\mathrm{P}_{0}$ is called as Compton momentun of a static particle.

This matter wave described in the above equation is a matter wave corresponding to the static particle associated with B system, whose wavelength $\lambda_{0}$ and $v_{0}$ reflect the space-time scale of the static particle. Here the particle is no longer a material point, but has a certain space-time scale, which corresponds to a circular flow with a radius of $\lambda_{0}$. If we consider the special case of an electron spin as $\mathrm{h} / 2$, then the electron can only restore the original state after two times of rotations $(4 \pi)$, so the electron as the circulation is a Mobius strip. The $\varphi_{0}$ is the space-time background field in A system and B system, when the particle does not move.

In the view of A system, the particle appears to be in constant linear motion, so the particle's mass changes from $\mathrm{m}_{0}$ to $\mathrm{m}, \mathrm{m}=\mathrm{m}_{0}\left(1-\mathrm{v}^{2} / \mathrm{c}^{2}\right)^{-1 / 2}$. According to quantum mechanics, the frequency of fluctuations associated with the particle is $v$ ${ }_{2}=\mathrm{mc}^{2} / \mathrm{h}$. 
If Compton wavelength of a moving particle is defined as $\lambda_{c}=h / m c$, then $v_{2}$ and $\lambda_{c}$ constitute a Compton matter wave $\varphi$ associated with a moving particle in A system: $\varphi=a \bullet \exp \left\{(2 \pi \mathrm{i} / \mathrm{h})\left(\mathrm{P}_{\mathrm{c}} \bullet \mathrm{r}-\mathrm{Et}\right)\right\}$

In this formula, $\mathrm{Pc}=\mathrm{mc}, \mathrm{E}=\mathrm{mc}^{2}$, $\mathrm{Pc}$ also has the momentum characterist, $\mathrm{so} \mathrm{Pc}=\mathrm{mc}$ is called as Compton momentum of a moving particle, and $\mathrm{c}$ is the wave velocity. $\varphi$ represents the space-time background information of quantum inner fluctuations of a moving particle.

Then let's assume that the wave $\varphi_{0}$ that corresponds to a static particle completes a total oscillation time is T. When the particle is at rest, the stationary observer in B system uses $\tau_{0}$ as a time unit, he measures the period of vibration of the wave field and gets it as follow : $\mathrm{T}_{0}=\mathrm{T} / \tau_{0}$

Therefore, the frequency of the wave field corresponding to a particle at rest is:

$$
v_{0}=1 / \mathrm{T}_{0}=\tau_{0} / \mathrm{T} \text {. }
$$

When the particle is moving, a static observer in A system looks at the fluctuation associated with the particle, his units that measure time becomes bigger: $\tau_{0}{ }^{\prime}=\tau_{0}\left(1-\mathrm{v}^{2} / \mathrm{c}^{2}\right)^{-1 / 2}$, so the period of $\varphi$ with the wave field corresponding to the moving particle becomes this formular: $\mathrm{T}_{0}{ }^{\prime}=\mathrm{T} / \tau_{0}{ }^{\prime}$. The frequency of the corresponding wave field $\varphi$ after the particle is moving: $v^{2}=1 / \mathrm{T} 0^{\prime}=\tau 0^{\prime} / \mathrm{T}$.

So the relationship between $v_{0}$ and $v_{2}$ is: $v_{0} / v_{2}=\left(\tau_{0} / \mathrm{T}\right) /\left(\tau_{0}{ }^{\prime} / \mathrm{T}\right)=\tau_{0} / \tau_{0}{ }^{\prime}=\left(1-\mathrm{v}^{2} / \mathrm{c}^{2}\right)^{1 / 2}$,

And $v_{2}=v_{0}\left(1-v^{2} / c^{2}\right)^{-1 / 2}$

This is consistent with the results from the mass-energy relationship and Planck formula, but its physical meaning is clearer. $v_{0}$ is the field frequency of the static Compton matter wave $\varphi_{0}$ when the particle is at rest, $v_{0}$ is the field frequency of the corresponding dynamic Compton matter wave $\varphi$ when the particle is moving. Obviously, the frequency of Compton matter wave corresponding to the moving particle is higher, it's not inconsistent with decreasing frequency of a moving clock, and the two are causal each other: It is because a moving particle's inner clock has a low frequency, then its Compton matter wave frequency increases, and Compton matter wave is a matter wave field measured by a light clock. This naturally solves the paradox of the two unequal frequencies in De Broglie's phase wave theory, and uses a same Lorentz frame of reference.

If the measured length unit of the atomic clock in a moving system becomes larger, the length of the measured object produces Lorentz contraction effect, we can also infer that Compton matter wave length of a moving particle is smaller than Compton matter wave length of a stationary particle. It is safe to say that the matter wave corresponds to a moving particle what De Broglie refers to should be a moving Compton matter wave rather than the original phase wave. In the new model of Zhao Guoqiu, the particle is conceived as a circular flow in the speed of light, and the velocity of Compton matter wave is also the speed of light. Whether in the stationary or the moving system, the frequency and phase of the particle circulation which is measured by light signal static relative to the particle itself, is consistent with frequency and phase of Compton matter wave.

Let's take a look at the relationship between Compton matter wave and Schrodinger matter wave.

Starting from relativistic energy relations: $E^{2}=p^{2} c^{2}+E_{0}^{2}\left(m c^{2}\right)^{2}=(m v)^{2} \cdot c^{2}+m_{0}^{2} c^{4}$, Divided the following equation by $c^{2}$, and you get $(\mathrm{mc})^{2}=(\mathrm{mv})^{2}+\left(\mathrm{m}_{0} \mathrm{c}\right)^{2}$.

Let $\mathrm{p}_{1}=\mathrm{mv}$, and $\mathrm{p}_{1}$ is the relativistic momentum of a moving particle.

So $\mathrm{p}_{\mathrm{c}}{ }^{2}=\mathrm{p}_{1}^{2}+\mathrm{p}_{0}^{2}$.

The stationary Compton momentum $\mathrm{p}_{0}$, the moving Compton momentum $\mathrm{p}_{\mathrm{c}}$, and the relativistic momentum $\mathrm{p}_{1}$ form a right triangle, we express it in vectors as follows: $\mathrm{p}_{\mathrm{c}}=\mathrm{p}_{1}+\mathrm{p}_{0}$

There is a small angle between $\mathrm{p}_{\mathrm{c}}$ and $\mathrm{p}_{0}$, while $\mathrm{p}_{1}$ is in the same direction as $\mathrm{r}$ of a moving electron's displacement ( $\mathrm{p}_{1}$ is a projection of $p_{c}$ in $r$ direction). Therefore, $p_{c}$ can produce an observable fluctuation effect of the particle through $p_{1}$ in its space direction. A matter wave with $\mathrm{p}_{1}$ is observable, so it is Schrodinger matter wave when $\mathrm{m}=\mathrm{m}_{0}$. Taking into account the relativistic effect, $\mathrm{p}_{1}$ and E forms Dirac matter wave, and it has observable effects which the electron's eigenmomentum mc shows its relativistic momentum mv by its zigzag effects. However, the wavelength of the two matter waves does not represent the size of the particle, it can only contribute to the change in the size of the particle, because the actual size of an electron should be determined by Compton momentum $\mathrm{p}_{\mathrm{c}}$, it is an eigenmomentum solution of the Dirac equation. $\mathrm{p}_{1}$ is just a projection of $\mathrm{p}_{\mathrm{c}}$ in the direction of motion, and another projection perpendicular to the direction of motion is the intrinsic momentum $\mathrm{p}_{0}$.

Zhao Guoqiu connects the wavelength of Compton matter wave with the radius of the particle circulation, and then the surface image of the particle circulation in space is represented by quantum curvature, he finds that quantum curvature from the normalized wave function is proportional to quantum probability, but it includes more qualitative characteristics of quantum fluctuations of a particle, this is the phase information expressed by the direction of a surface, it has to do with intrinsic momentum direction of a particle (static Compton momentum). The information propagation speed of Compton matter wave associated with a particle's intrinsic quantum fluctuations is the speed of light c, but the moving wave image velocity associated with the particle's extrinsic motion is $\mathrm{u}=\mathrm{c}^{2} / \mathrm{v}$, this is a phase wave that excludes direct transfer of momentum and energy.

In special relativity, we can consider how the velocity superposition principle of particle motion is different from the parallelogram law of velocity vector superposition in Newtonian mechanics. Shi zhiheng believes that the principle of velosity superposition of relativity is also fit for De Broglie phase wave. 
Because the phase velocity of matter wave $\left(m_{0} \neq 0\right)$ is, its Doppler effect formula cannot imitate the formula of light wave. Shi Zhiheng assumes that $\mathrm{K}(\mathrm{x}, \mathrm{t})$ system is moving relative to $\mathrm{K}^{\prime}\left(\mathrm{x}^{\prime}, \mathrm{t}^{\prime}\right)$ system in velosity $\mathrm{v}$ ', and $\mathrm{v}$ ' is the same direction as $\mathrm{v}$, then he gets formulas in view of $\mathrm{K}^{\prime}\left(\mathrm{x}^{\prime}, \mathrm{t}^{\prime}\right)$ system(Let's say the starting point is the origin) as follows:

$\square x \square \square \square \square(x \square \square \square \square t)$

$$
\left\{\begin{array}{l}
x=\beta^{\prime}\left(x^{\prime}-v^{\prime} t^{\prime}\right) \\
t=\beta^{\prime}\left(t^{\prime}-\frac{v^{\prime}}{c^{2}} x^{\prime}\right)
\end{array}\right.
$$

Puts $\mathrm{x}, \mathrm{t}$ into $\psi(\mathrm{x}, \mathrm{t})$, Shi gets a formula as follows:

$$
\psi\left(x^{\prime}, t^{\prime}\right)=\exp \left\{i \omega \beta^{\prime}\left(1+\frac{v v^{\prime}}{c^{2}}\right)\left[t^{\prime}-\left(\frac{v^{\prime}+v}{1+\frac{v^{\prime} v}{c^{2}}} / c^{2}\right) x^{\prime}\right]\right\}
$$

So

$$
\left\{\begin{array}{l}
\omega^{\prime}=\omega \beta^{\prime}\left(1+\frac{v v^{\prime}}{c^{2}}\right)=\omega_{0} \beta \beta^{\prime}\left(1+\frac{v v^{\prime}}{c^{2}}\right)=\omega_{0} \beta^{\prime \prime} \\
v^{\prime}=v \beta^{\prime}\left(1+\frac{v v^{\prime}}{c^{2}}\right)=v_{0} \beta \beta^{\prime}\left(1+\frac{v v^{\prime}}{c^{2}}\right)=v_{0} \beta^{\prime \prime} \\
\beta^{\prime}=1 / \sqrt{1-v^{\prime 2} / c^{2}} \\
u^{\prime}=c^{2} / \frac{v+v^{\prime}}{1+\frac{v v^{\prime}}{c^{2}}}=\omega^{\prime} / k^{\prime} \\
u_{g}^{\prime}=\frac{u_{g}+v^{\prime}}{1+\frac{v v^{\prime}}{c^{2}}}=\frac{v+v^{\prime}}{1+\frac{v v^{\prime}}{c^{2}}}
\end{array}\right.
$$

Among them,

$$
\beta^{\prime \prime}=\beta \beta^{\prime}\left(1+\frac{v v^{\prime}}{c^{2}}\right)=1 / \sqrt{1-u_{g}^{\prime 2} / c^{2}}
$$

But if we put $\exp i \omega\left(t-\frac{x}{u}\right)$ into Lorentz transformation and get $\exp i \omega^{\prime}\left(t^{\prime}-\frac{x^{\prime}}{u^{\prime}}\right)$

Then

$$
\left\{\begin{array}{l}
\omega^{\prime}=\beta^{\prime} \omega\left(1+\frac{v^{\prime}}{u}\right) \\
\frac{\omega^{\prime}}{u^{\prime}}=\beta^{\prime} \frac{\omega}{u}\left(1+\frac{u v^{\prime}}{c^{2}}\right)
\end{array}\right.
$$

We may obtain

$$
u^{\prime}=\frac{u+v^{\prime}}{1+u v / c^{2}}=c^{2} / \frac{c^{2} / v^{2}+c^{2} / v^{\prime}}{1+\frac{c^{2}}{v} \frac{c^{2}}{v^{\prime}} / c^{2}}
$$

And this superposition equation of the phase velocity is exactly the same as that of the particle velocity synthesis equation. This derivation is also applicable to the phase velocity of light $:(u \neq c)$ in the medium, and we compare it with the modified formulas of the famous Fresnel-Fizeau experiment.

\section{The development of dual 4-dimensional complex space-time description of quantum curvature}

In his 2013 paper, Zhao Guoqiu conceived the dual 4 dimensional spacetime representation of quantum curvature, and pointed out: 'The 4 dimensional space structure of the microscopic object itself is described by a set of imaginary curvature coordinates $\mathrm{K}_{1}, \mathrm{~K}_{2}, \mathrm{~K}_{3}$ and $\mathrm{K}_{4}$ of the mapping space of the Dual 4 dimensional complex space-time----- the Dual 4 dimensional complex phase space. The position of the microscopic object in the real space-time is described by the real coordinate $\mathrm{x}_{1}, \mathrm{x}_{2}, \mathrm{x}_{3}$ and $\mathrm{x}_{4}$ of the dual 4 dimensional complex space-time, the imaginary part of the curvature of 
the coordinates $\mathrm{K}_{2}, \mathrm{~K}_{3}, \mathrm{~K}_{4}$ correspond respectively to the real coordinates $\mathrm{x}_{2}, \mathrm{x}_{3}, \mathrm{x}_{4}$ and $\mathrm{K}_{1}=\mathrm{mc} / \mathrm{h}$, corresponds to $\mathrm{x}_{1}=$ ct.' $[4, \mathrm{p} 148]$.

Zhao Guoqiu introduces the curvature $\mathrm{K}$ related to Compton momentum, and Compton momentums satisfy the right triangle relation of the orthogonal vector $(1+3)$ decomposition in relativity : $m c=m_{0} c+m v$. This is an extension of the concept of wave vector (wave number), and realizes a geometric description of the quantum wave function, where $\mathrm{K}_{0}=$ $1 / \mathrm{r}_{0}=\mathrm{m}_{0} \mathrm{c} / \mathrm{h}$ is the curvature of the phase circle of the compton wavelength of the stationary particle, representing the bending of a great circle path on the rotational field ball associated with the spin of a particle. And $\mathrm{K}_{1}=\mathrm{mc} / \mathrm{h}$ is the curvature of a phase circle of Compton wavelength of a moving particle, $K_{(2,3,4)}$ is 3 projections of $K_{1}$ in the three directions of space dimensions, which is related to classical momentum mv. [4,p149] Quantum mechanics in dual 4dimensional spacetime modifies the orthodox measurement hypothesis, this new interpretation considers that quantum measurement is both a transition from microscopic discontinuity to macroscopic continuum, and an experimental operation process of eliminating catastrophe areas, discontinuity and the fixed phases, destroying orthogonality, destroying linearity among quantum eigenstates, and then restoring macroscopic chronology and causality.[5,p162]

In this way, theoretical structure of quantum mechanics shows an intrinsic relationship between special relativity and general relativity through the analysis of space-time dynamics. Firstly, Zhao Guoqiu seeks to understand the state superposition principle of quantum mechanics as a Fourier expansion of a stationary wave function, which can be used to establish an inertial frame in an eigenstate of each momentum, and the parallel coexistence of the eigenstates correspond to a set of parallel coexistence of inertial frame groups. Lorentz transformation of a wave function is equivalent to a simultaneous transformation of all eigenstates in a set of parallel inertial frame groups.[5,p161-162] Therefore, quantum mechanics essentially divides continuous actions of eigenstates and continuous motions of a physical process into quantum segmentations, and cuts off macroscopic causal realtions and become a series of discontinuous uniform motions (This is equivalent to taking a macroscopic continuous interaction into a series of electrons in atomic orbital transitions, electron collisions, and Compton effects of scattered photons in quantum electrodynamics ). An alteration from one eigenstate to another is equivalent to an alteration from one inertia system to another, the phase wave with different engenmomentums has different space-time origins, leading to chronology destruction. The quantum state transition between two electron orbits in the atom is similar to the coordinate transformation between two inertial systems. [4,p161] Quantum measurement process recovers from the superposition of the different eigenstates of microscopic objects of the quantum mechanics to a macroscopic continuous mechanical state

corresponding to microscopic objects. This involves restoring continuous interaction, eliminating orthogonal quantization partition, destroying linear superposition, and restoring macroscopic causality. Quantum measurement results in a transition from microscopic dual 4 dimensional complex space-time to macroscopic 4 dimensional real space-time, from field matter sphere to point particle, from matter wave to probability wave, from microscopic discontinuous actions to macroscopic continuous actions.[5,p162]

Secondly, starting from the quantization of energy and momentum, and resuming the macroscopic continuous action, physical conditions of the linear superposition of the wave function will be destroyed: On the one hand, due to the coexistence of eigenstates, the superposition state does not exist so that independent coherent sources lost physically, and self-coherences disappear. This is a physical reason why macroscopic objects can't be self coherent. On the other hand, energy and momentum quantization disappears, continuous forces restore. Various eigenstates' inertial systems associated with free microscopic objects describerd earlier become accelerated systems, a microscopic object will also be reduced to a whole(particle) acceleration state from its eigenstates superposition, the inertial force ( or equivalent gravity ) appears. When an inertial frame becomes a Non - inertial frame, its space becomes uneven, and Lorentz covariance becomes invalid.' [5,p161-162] This means that quantum origin of an inertial force is accompanied by a transition from eigenstates' inertail systems to a macroscopic accelerated system.

Thirdly, if the construction of special relativity includes transforming space-time characteristics of a moving object itself into its coordinate system's space-time attributes, and ignores its space form structure and abstract process that simplifies it to a particle; then in the microscopic field, a matter sphere of the rotational field can be obtained by an inverse process that change a particle into a quantum fluctuation in the dual 4-dimensional complex space-time through the relativistic space - time attribute of coordinate system, and the matter wave equation of De Broglie - Schrodinger can be deduced. The amplitude and phase of the wave function contains velocity information, geometric structure information and matter density information of a microscopic object, indicating that it is not a 'particle', and its hidden freedom of space is expanded in the dual 4 - dimensional space-time.[5,p176]

However, Zhao Guo-qiu's analysis of the inertial and accelated systems about the superposition principle of the quantum wave function, is difficult to be extended to study in quantum gravity theory. First of all, because there is no quantum gravity equation like Schodinger or Dirac equation can give a quantum gravity stationary state in the general case. Secondly, because general relativity is nonlinear, many difficulties appears when a stationary wave function of quantum gravity makes a Fourier expansion. If there is an automatic decoherence process that an atom's orbital energy transition decreases when its orbital energy level increases in quantum mechanics, then the mechanism of gravitation is not clear in which level of matter structure that quantum gravity can transition to continuous curved space - time. So far the so-called quantum gravity energy level transition is only a theoretical hypothesis, because there is no orbital energy level model like atomic structure model at the Planck time scale. Finally, ( $1+3)$ momentum decomposition of Compton matter wave in 4 - dimensional spacetime requires the existence of orthogonal space - time frame, so that there are still enormous mathematical physical difficulties when we combine quantum mechanics curvature interpretation in the dual 4 dimensional complex space-time frame with curved space-time frame of general relativity. 


\section{[References]}

[1].Jin Shangnian, The physical foundation and philosophical background of quantum mechanics[M], Fudan university press, Shanghai, China,2007.

[2].Albert Einstein and others, The principle of relativity theory[M], Chinese version by Zhao Zhitian and Liu Yiguan, Science press, Beijing, China, 1980.

[3].Zhao Guoqiu,Gui Qiquan,Wu Xinzhong and Wan Xiaolong, The new melody of physics-----Quantum mechanics curvature interpretation[M], Wuhan press, Wuhan, China, 2004.

[4].Zhao Guoqiu, Quantum mechanics description of dual 4 - dimensional space - time (Quantum mechanics curvature interpretation) [J], pubished in Modern Physics, Volume 3, Number 5, November 2013, Hans Publisher, p147-160.

[5].Zhao Guoqiu,Quantum Mechanics Foundation in Dual 4-dimensional space-time -------The Space-time origins of quantum probability[M], Hubei science and technology press, Wuhan, China,2016. 